Check for updates

Cite this: Chem. Commun., 2018, 54, 13096

Received 31st July 2018

Accepted 19th September 2018

DOI: $10.1039 / \mathrm{c} 8 \mathrm{cc} 06253 f$

rsc.li/chemcomm

\section{Template-promoted self-replication in dynamic combinatorial libraries made from a simple building block $\dagger$}

\author{
B. Bartolec, M. Altay and S. Otto (D) *
}

\begin{abstract}
We report dynamic combinatorial libraries made from a simple building block that is on the verge of enabling self-assembly driven self-replication. Adding a template provides a sufficient additional push yielding self-replication. Self-assembly and self-replication can emerge with building blocks that are considerably smaller than those reported thus far.
\end{abstract}

Dynamic combinatorial libraries (DCLs) ${ }^{1}$ are attracting increasing attention as a powerful tool in the emerging field of systems chemistry. ${ }^{1 b, 2}$ DCLs allow the discovery and development of systems that exploit non-covalent interactions. In a DCL the library members interconvert continuously by exchanging building blocks with each other, leading to an (equilibrium) pool of interchanging molecules. Non-covalent interactions tend to stabilize those library members that are most efficient at forming them, causing the library composition to shift in favour of these compounds. Such non-covalent interactions can occur within or between library members, giving rise to foldamers ${ }^{3}$ or self-assembling systems, ${ }^{4}$ respectively. Dynamic combinatorial self-assembly processes can give rise to self-replication, ${ }^{5,6}$ producing self-synthesizing materials ${ }^{4 b, 7}$ in an autocatalytic fashion. Upon addition of a template DCLs may also produce molecules or nanoparticles that selectively bind to this template. Molecular recognition between the template and library species leads to the amplification of the library members which bind to the template. This effect may be utilized for the discovery of ligands for biomacromolecules, ${ }^{8}$ surface-functionalised nanoparticles, ${ }^{9}$ synthetic receptors ${ }^{10}$ and even catalysts. ${ }^{11}$ On rare occasions templates can also induce assembly processes and trigger the emergence of a self-replicator from a dynamic combinatorial library. ${ }^{12}$

The criteria for the design of building blocks to generate DCLs that efficiently exploit non-covalent interactions are still relatively obscure, in particular when it comes to self-assembly

Centre for Systems Chemistry, Stratingh Institute, University of Groningen, Nijenborgh 4, 9747 AG Groningen, The Netherlands. E-mail: s.otto@rug.nl $\dagger$ Electronic supplementary information (ESI) available: Building block synthesis, supplementary figures and UPLC-MS data. See DOI: 10.1039/c8cc06253f and replication phenomena. Past work on self-assembly driven self-replication has mostly featured relatively elaborate peptide building blocks. ${ }^{4 b, 6 a, b, 13}$ We now show that a building block containing only a single amino acid has already a tendency to produce a self-replicator and that this process can be promoted by addition of a template molecule.

The design of new building blocks for DCLs aimed at selfassembly involves a balancing act between two requirements: firstly, building blocks need to endow library members with the possibility to form attractive intermolecular interactions and secondly, building block and library members should be soluble and therefore have attractive interactions with the solvent. When targeting selfassembly in water the latter typically requires the presence of charged or ethylene oxide groups. We designed building block 1 (Scheme 1) as a structurally simple (minimal) building block that features a carboxylate for water solubility, two hydrophobic aromatic rings for hydrophobic interactions and an amide group as a potential hydrogen bonding site. It also contains two thiol groups which can be oxidized to form a DCL of macrocyclic disulfides when exposed to oxygen from the air. ${ }^{14}$ At neutral to mildly basic $\mathrm{pH}$ residual thiolate mediates the disulfide exchange reaction that enables the equilibration of the different library members.

We set up a DCL made by dissolving 1 at a $3.0 \mathrm{mM}$ concentration in aqueous borate buffer (50 mM, pH 8.5) and monitored the product distribution by UPLC-MS. A diverse set of oligomers is formed, ranging from cyclic trimer to cyclic $16^{\text {mer }} \downarrow^{15}$
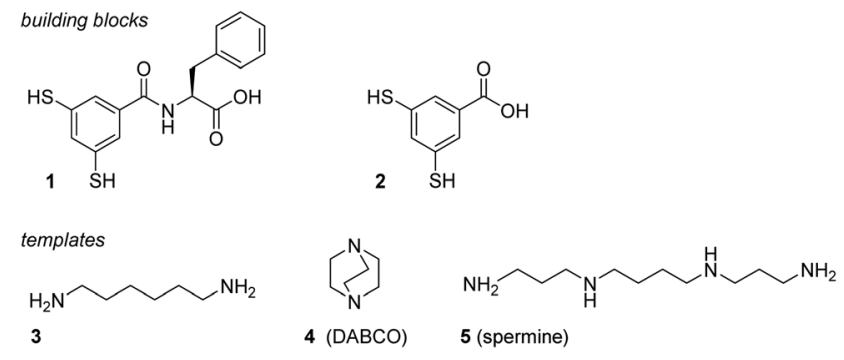

Scheme 1 Structures of building blocks and templates. 


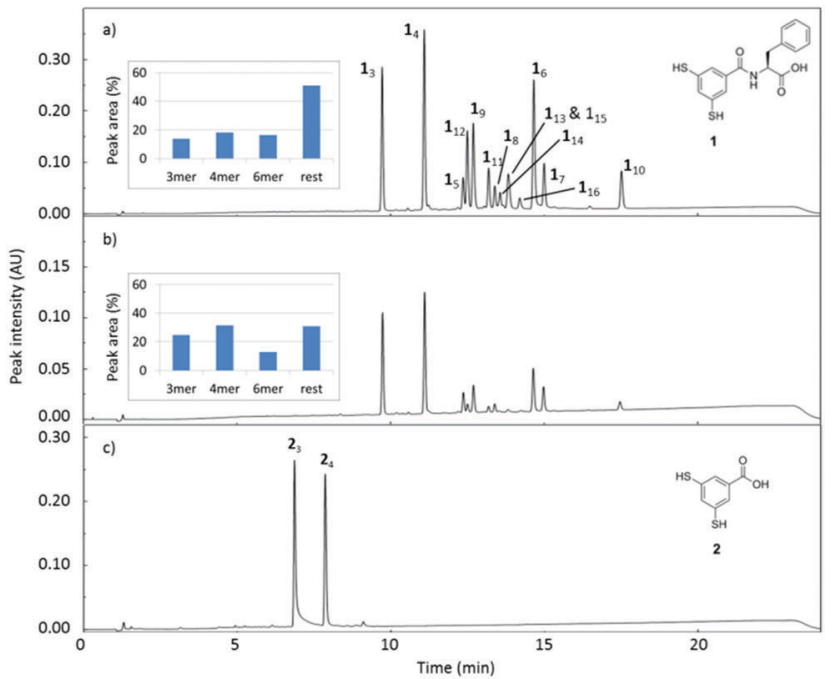

Fig. 1 UPLC-MS analysis of 50 days old DCLs made from building block 1 in aqueous borate buffer $(50 \mathrm{mM}, \mathrm{pH} 8.5)$, stirred at $1200 \mathrm{rpm}$ at room temperature: (a) $[\mathbf{1}]=3.0 \mathrm{mM}$; (b) $[\mathbf{1}]=1.0 \mathrm{mM}$. The inserts emphasise the higher percentage of larger oligomers at higher concentrations. (c) UPLCMS analysis of a DCL made from control compound $\mathbf{2}$ under the same conditions $([2]=1.0 \mathrm{mM}) \neq$

This DCL is considerably more diverse than that formed from control compound $\mathbf{2}$ which is an analogue of $\mathbf{1}$ but lacking the phenylalanine residue (Fig. 1c). The latter DCL consisted predominantly of cyclic trimer and tetramer.

Inspection of TEM micrographs revealed ill-defined supramolecular aggregates in DCLs made from building block 1 (Fig. S1, ESI $\dagger$ ), while no aggregates were observed in DCLs made from building block 2. Thus, it appears that the introduction of a single phenylalanine residue in $\mathbf{1}$ is sufficient to allow for the formation of self-assembled structures. Such assembly may also explain the formation of larger macrocycles. ${ }^{15}$ In the assemblies there is a high local concentration of material that enables the formation of relatively high molecular weight macrocycles through disulfide exchange. In DCLs of non-assembling library members, such as those made from 2, the formation of such large oligomers tends to be entropically disfavoured, hence only the smallest non-strained rings are formed (trimer and tetramer). \& Note that in DCLs made from 1 at lower concentrations (Fig. 1b) the larger macrocycles are less populated, suggesting that the assembled structures are at equilibrium with non-assembled structures in the concentration range that was probed. This observation, combined with the ill-defined nature of the aggregates (Fig. S1, ESI $\dagger$ ) suggests that the design of building block $\mathbf{1}$ is only just good enough to lead to self-assembled library members. As assembly most likely comes about through a trade-off between attractive interactions (hydrogen bonding and hydrophobically enhanced $\pi$-stacking interactions) and electrostatic repulsion between the carboxylate groups, we reasoned that it might be possible to promote assembly by adding a template that could shield charge repulsion. Thus, amines 3-5 were tested for their ability to direct self-assembly. The linear primary amines $\mathbf{3}$ and $\mathbf{5}$ induced a template effect, selectively promoting the formation of the cyclic hexamer, while virtually no effect was observed with secondary amine 4 (Fig. 2).
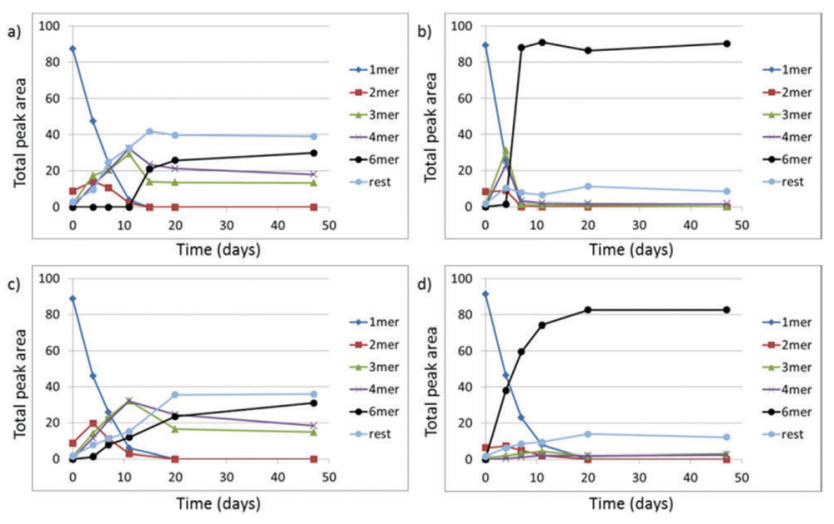

Fig. 2 Change of the product distribution with time for DCLs made from building block $1(1.0 \mathrm{mM}$ in $50 \mathrm{mM}$ borate buffer, $\mathrm{pH} 8.5$, stirred at $1200 \mathrm{rpm}$, room temperature) in the presence of different templates (1.0 mM): (a) no template; (b) 3; (c) DABCO, 4; (d) spermine, 5.

We speculate that hydrogen bonding between primary amine groups of the template molecules and carboxylate groups present in library members causes the observed template effect, although we cannot exclude that the close proximity between the two amines in $\mathbf{4}$ or steric hindrance provided by the ethylene bridges would preclude efficient interaction with the fibres.

The extent to which the formation of $\mathbf{1}_{6}$ is autocatalytic was probed by seeding experiments. A small amount of $\mathbf{1}_{6}$ was added to a library composed of different oligomers, containing only a low amount of the suspected replicator. The rate of growth of $\mathbf{1}_{6}$ was monitored and clearly faster than the corresponding rate in the absence of seed, confirming that $\mathbf{1}_{6}$ is a self-replicator (Fig. 3). This is in agreement with the sigmoidal growth curve of $\mathbf{1}_{6}$ in the absence of seed (Fig. 2a). Note that $\mathbf{1}_{6}$ is the only macrocycle to show such sigmoidal growth. The latter two observations suggest that $\mathbf{1}_{6}$ can also self-replicate in the absence of a template.

Self-replication of $\mathbf{1}_{6}$ is driven by self-assembly of this macrocycle into fibrous structures that were visualised by TEM (Fig. 4). In a library prepared without template, isolated fibres were observed suggesting a simple one-dimensional self-assembly mode (Fig. 4a). In the presence of the amine templates $\mathbf{3}$ or $\mathbf{5}$ laterally associated fibres were observed (Fig. $4 \mathrm{~b}$ and c), suggesting that the templates, by partially compensating the negative charge of the fibres, reduce charge repulsion allowing fibres to assemble into larger aggregates.

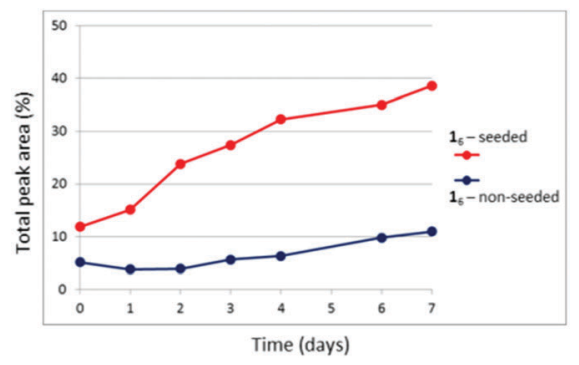

Fig. 3 Seeding experiment for assessing the ability of $\mathbf{1}_{6}$ to self-replicate. A library of building block 1 (10\% volume) containing approximately $90 \%$ of hexamer (Fig. 2b, day 20) was added to an $80 \%$ oxidized DCL made from building block 1 (1.0 mM, stirred at $1200 \mathrm{rpm}$, room temperature). 


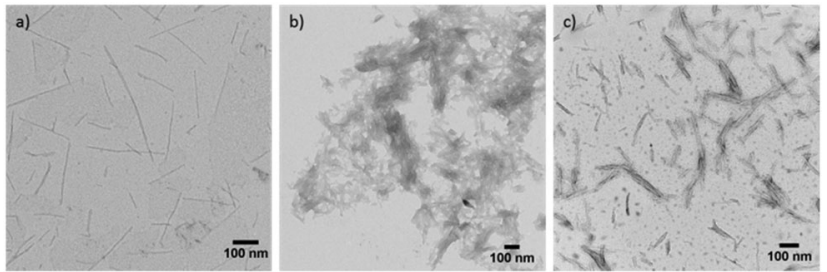

Fig. 4 Representative TEM micrographs of 15 days old DCLs made from building block $1(1.0 \mathrm{mM}$ in $50 \mathrm{mM}$ borate buffer, $\mathrm{pH} 8.5$, stirred at $1200 \mathrm{rpm}$, room temperature) (a) in the absence of template; (b) in the presence of $20 \mathrm{~mol} \% \mathbf{3}$; (c) in the presence of $20 \mathrm{~mol} \% \mathbf{5}$. Corresponding library compositions are shown in Fig. 3 and Fig. 2a, b, d, respectively.

Further confirmation that building block 1 yields DCLs that are on the border of allowing access to self-assembling molecules was obtained by experiments in which we mixed this building block with analogue 2 . The resulting DCL consisted of small trimeric and tetrameric macrocycles of mixed composition, irrespective of the addition of template (Fig. S3, ESI $\dagger$ ), suggesting that no significant self-assembly takes place in these mixed DCLs.

In conclusion, we have identified a minimal building block design which can produce DCLs with just enough potential for intermolecular non-covalent interactions to facilitate selfassembly. Unassisted by templates this building block produces a diverse mixture of macrocycles. In the presence of suitable primary amine templates a self-replicating cyclic hexamer is formed selectively, driven by assembly into fibres. These results show that self-replication can occur with surprisingly simple building blocks, much less complex than the relatively long peptides used in most studies thus far, ${ }^{4 b, 6 a, b, 13,16}$ showing the generality and potential of self-assembly driven self-replication.

We are grateful for support from the European Research Council (ERC advanced grant no. 741774 - ToDL), the NWO (grant no. VICI - 724.012.002) and the Dutch Ministry of Education, Culture and Science (Gravitation program 024.001.035). Reference spectroscopic and reaction data can be found at DOI: 10.1039/c8cc06253f.

\section{Conflicts of interest}

There are no conflicts to declare.

\section{References}

¥ Peak areas can be taken to represent concentrations (expressed in units of building blocks) as control experiments showed that the total peak area observed for DCLs with substantially different compositions is approximately constant, indicating that the building block unit has a molar absorptivity that is essentially independent of the size of the macrocycle in which it resides.

$\S$ We cannot exclude that also non-covalent interactions within the macrocycles lead to the stabilization of larger rings. The fact that UPLC peak area does not fall off gradually with increasing ring size suggests that specific ring sizes may benefit from such intramolecular stabilisation.

1 (a) D. Komaromy, P. Nowak and S. Otto, in Dynamic Covalent Chemistry: Principles, Reactions, and Applications, ed. W. Zhang and Y. Jin, Wiley, Hoboken, 1997, ch. 2, pp. 31-120; (b) O. S. Miljanic, Chem, 2017, 2, 502-524; (c) Y. H. Jin, C. Yu, R. J. Denman and W. Zhang, Chem. Soc. Rev., 2013, 42, 6634-6654; (d) J. M. Lehn, Angew. Chem., Int. Ed., 2013, 52, 2836-2850; (e) F. B. L. Cougnon and J. K. M. Sanders, Acc. Chem. Res.,
2012, 45, 2211-2221; $(f)$ P. T. Corbett, J. Leclaire, L. Vial, K. R. West, J.-L. Wietor, J. K. M. Sanders and S. Otto, Chem. Rev., 2006, 106, 3652-3711.

2 (a) G. Ashkenasy, T. M. Hermans, S. Otto and A. F. Taylor, Chem. Soc. Rev., 2017, 46, 2543-2554; (b) E. Mattia and S. Otto, Nat. Nanotechnol., 2015, 10, 111-119; (c) K. Ruiz-Mirazo, C. Briones and A. de la Escosura, Chem. Rev., 2014, 114, 285-366; (d) J. R. Nitschke, Nature, 2009, 462, 736-738; (e) R. F. Ludlow and S. Otto, Chem. Soc. Rev., 2008, 37, 101-108.

3 (a) L. Roy and M. A. Case, J. Am. Chem. Soc., 2010, 132, 8894-8896; (b) Y. Krishnan-Ghosh and S. Balasubramanian, Angew. Chem., Int. Ed., 2003, 42, 2171-2173; (c) K. Oh, K. S. Jeong and J. S. Moore, Nature, 2001, 414, 889-893; (d) M. A. Case and G. L. McLendon, J. Am. Chem. Soc., 2000, 122, 8089-8090.

4 (a) A. Pal, M. Malakoutikhah, G. Leonetti, M. Tezcan, M. ColombDelsuc, V. D. Nguyen, J. van der Gucht and S. Otto, Angew. Chem., Int. Ed., 2015, 54, 7852-7856; (b) J. M. A. Carnall, C. A. Waudby, A. M. Belenguer, M. C. A. Stuart, J. J. P. Peyralans and S. Otto, Science, 2010, 327, 1502-1506; (c) R. Nguyen, L. Allouche, E. Buhler and N. Giuseppone, Angew. Chem., Int. Ed., 2009, 48, 1093-1096.

5 For reviews on self-replication, see: (a) H. Duim and S. Otto, Beilstein J. Org. Chem., 2017, 13, 1189-1203; (b) T. Kosikova and D. Philp, Chem. Soc. Rev., 2017, 46, 7274-7305; (c) G. Clixby and L. Twyman, Org. Biomol. Chem., 2016, 14, 4170-4184; (d) A. J. Bissette and S. P. Fletcher, Angew. Chem., Int. Ed., 2013, 52, 12800-12826; (e) V. Patzke and G. von Kiedrowski, ARKIVOC, 2007, 5, 293-310.

6 For recent examples on self-replicators from dynamic combinatorial libraries, see: (a) M. Altay, Y. Altay and S. Otto, Angew. Chem., Int. Ed., 2018, 57, 10564-10568; (b) Y. Altay, M. Tezcan and S. Otto, J. Am. Chem. Soc., 2017, 139, 13612-13615; (c) J. W. Sadownik, T. Kosikova and D. Philp, J. Am. Chem. Soc., 2017, 139, 17565-17573; (d) T. Kosikova and D. Philp, J. Am. Chem. Soc., 2017, 139, 12579-12590.

7 N. Giuseppone, Acc. Chem. Res., 2012, 45, 2178-2188.

8 For recent examples, see: (a) J. Soubhye, M. Gelbcke, P. Van Antwerpen, F. Dufrasne, M. Y. Boufadi, J. Neve, P. G. Furtmuller, C. Obinger, K. Z. Boudjeltia and F. Meyer, ACS Med. Chem. Lett., 2017, 8, 206-210; (b) L. Monjas, L. Swier, I. Setyawati, D. J. Slotboom and A. K. H. Hirsch, ChemMedChem, 2017, 12, 1693-1696; (c) J. Fu, H. X. Fu, M. Dieu, I. Halloum, L. Kremer, Y. F. Xia, W. D. Pan and S. P. Vincent, Chem. Commun., 2017, 53, 10632-10635.

9 (a) W. Edwards, N. Marro, G. Turner and E. R. Kay, Chem. Sci., 2018, 9, 125-133; (b) P. Nowak, V. Saggiomo, F. Salehian, M. ColombDelsuc, Y. Han and S. Otto, Angew. Chem., Int. Ed., 2015, 54, 4192-4197; (c) F. della Sala and E. R. Kay, Angew. Chem., Int. Ed., 2015, 54, 4187-4191; (d) Y. Han, P. Nowak, M. Colomb-Delsuc, M. P. Leal and S. Otto, Langmuir, 2015, 31, 12658-12663.

10 For recent examples, see: (a) F. Ulatowski and J. Jurczak, Pure Appl. Chem., 2017, 89, 801-807; (b) B. C. Peacor, C. M. Ramsay and M. L. Waters, Chem. Sci., 2017, 8, 1422-1428; (c) J. Atcher, J. Sola and I. Alfonso, Org. Biomol. Chem., 2017, 15, 213-219; (d) J. Li, P. Nowak and S. Otto, Angew. Chem., Int. Ed., 2015, 54, 833-837.

11 (a) H. Fanlo-Virgos, A. N. R. Alba, S. Hamieh, M. Colomb-Delsuc and S. Otto, Angew. Chem., Int. Ed., 2014, 53, 11346-11350; (b) L. Vial, J. K. M. Sanders and S. Otto, New J. Chem., 2005, 29, 1001-1003; (c) B. Brisig, J. K. M. Sanders and S. Otto, Angew. Chem., Int. Ed., 2003, 42, 1270-1273.

12 (a) D. Komaromy, M. Tezcan, G. Schaeffer, I. Maric and S. Otto, Angew. Chem., Int. Ed., 2017, 56, 14658-14662; (b) P. Nowak, M. Colomb-Delsuc, S. Otto and J. Li, J. Am. Chem. Soc., 2015, 137, 10965.

13 (a) M. Malakoutikhah, J. J. P. Peyralans, M. Colomb-Delsuc, H. FanloVirgos, M. C. A. Stuart and S. Otto, J. Am. Chem. Soc., 2013, 135, 18406-18417; (b) B. Rubinov, N. Wagner, M. Matmor, O. Regev, N. Ashkenasy and G. Ashkenasy, ACS Nano, 2012, 6, 7893-7901; (c) B. Rubinov, N. Wagner, H. Rapaport and G. Ashkenasy, Angew. Chem., Int. Ed., 2009, 48, 6683-6686.

14 (a) S. P. Black, J. K. M. Sanders and A. R. Stefankiewicz, Chem. Soc. Rev., 2014, 43, 1861-1872; (b) S. Otto, R. L. E. Furlan and J. K. M. Sanders, J. Am. Chem. Soc., 2000, 122, 12063-12064.

15 D. Komaromy, M. C. A. Stuart, G. M. Santiago, M. Tezcan, V. V. Krasnikov and S. Otto, J. Am. Chem. Soc., 2017, 139, 6234-6241.

16 (a) J. Nanda, B. Rubinov, D. Ivnitski, R. Mukherjee, E. Shtelman, Y. Motro, Y. Miller, N. Wagner, R. Cohen-Luria and G. Ashkenasy, Nat. Commun., 2017, 8, 434; (b) Z. Dadon, N. Wagner, S. Alasibi, M. Samiappan, R. Mukherjee and G. Ashkenasy, Chem. - Eur. J., 2015, 21, 648-654; (c) D. H. Lee, J. R. Granja, J. A. Martinez, K. Severin and M. R. Ghadiri, Nature, 1996, 382, 525-528. 